\title{
TURNPIKE THEOREMS BY A VALUE FUNCTION APPROACH
}

\author{
Alain Rapaport $^{1}$ and Pierre Cartigny $^{2}$
}

\begin{abstract}
Turnpike theorems deal with the optimality of trajectories reaching a singular solution, in calculus of variations or optimal control problems. For scalar calculus of variations problems in infinite horizon, linear with respect to the derivative, we use the theory of viscosity solutions of HamiltonJacobi equations to obtain a unique characterization of the value function. With this approach, we extend for the scalar case the classical result based on Green theorem, when there is uniqueness of the singular solution. We provide a new necessary and sufficient condition for turnpike optimality, even in the presence of multiple singular solutions.
\end{abstract}

Mathematics Subject Classification. 34H05, 49K05, 49L25.

Received Januray 8, 2003. Revised August 6, 2003.

\section{INTRODUCTION}

In this paper we consider a problem of calculus of variations in infinite horizon whose objective $J[$.$] is given by:$

$$
J[x(.)]=\lim _{T \rightarrow+\infty} \int_{0}^{T} \mathrm{e}^{-\delta t} l(x(t), \dot{x}(t)) \mathrm{d} t,
$$

where $\delta$ is a positive number and $l$ is a real valued function on $\mathbb{R} \times \mathbb{R}$, linear w.r.t. his second argument. Our interest is the maximization of $J$ over the paths $x\left(\right.$.) with fixed initial condition $x(0)=x_{0}$, for which the velocities respect some inequality constraints (that will be made more precise later) and such that the improper integral (1) converges.

For such a problem, linear with respect to the velocity, the Euler first order optimality condition is no longer a differential equation but an algebraic one. Moreover as we will assume, the integrand $l(.,$.$) doesn't depend$ on the time, so for solutions $\bar{x}$ of this equation, the stationary paths $\bar{x}($.$) defined by:$

$$
\bar{x}(t)=\bar{x}, \quad \forall t,
$$

are the only $C^{1}$ candidates for optimality. Particularly, with this approach, we cannot say anything about hypothetical optimal solutions emanating from initial conditions $x_{0}$ that are not solutions of the algebraic Euler equation. On the other hand it is another difficulty to prove the optimality of these candidates, because the

Keywords and phrases. Calculus of variations, infinite horizon, Hamilton-Jacobi equation, viscosity solutions, turnpike.

1 UMR Analyse des Systèmes et Biométrie, 2, place Viala, 34060 Montpellier, France; e-mail: rapaport@ensam.inra.fr

2 GREQAM, université de la Méditerranée, 2, rue de la Vieille Charité, 13002 Marseille, France;

e-mail: cartigny@ehess.cnrs-mrs.fr 
problem is with infinite horizon. For this case only few results, like standard necessary or sufficient optimality conditions, are well established $[3,4,7]$. We can also underline that the approach via the Pontryagin Maximum principle causes analog difficulties, particularly with the use of the transversality condition [5]. For those reasons we propose here an approach via the value function and an associated Hamilton-Jacobi equation.

Before we detail our approach, we recall the existing results for this problem, concerning the so called turnpike property. In the literature, quite a few theoretical results exist. To our knowledge, they only concern the scalar case, with the Euler equation possessing an unique solution $\bar{x}$. More precisely, we consider the case of constrainted velocities given by:

$$
\alpha \leq \dot{x}(t) \leq \beta, \quad \forall t,
$$

where $\alpha<0$ and $\beta>0$ are two constants. Then for any $x_{0}$ it is relevant to introduce the Most Rapid Approach Path (MRAP) from $x_{0}$ to $\bar{x}$, which is the trajectory joining $x_{0}$ to $\bar{x}$ as quickly as possible (i.e. using either the velocity $\alpha$ or the velocity $\beta$ ). When MRAPs are optimal from any initial condition $x_{0} \in \mathbb{R}, \bar{x}$ is then called a turnpike.

The standard result is a sufficient condition under which $\bar{x}$ is a turnpike. It can be proved that the condition:

$$
(\bar{x}-x)\left(A^{\prime}(x)+\delta B(x)\right) \geq 0, \quad \forall x \in \mathbb{R},
$$

in addition to a technical assumption, is sufficient for the optimality of MRAPs. This result is established using a method introduced by Miele [10], based on Green theorem. Hartl and Feichtinger have proved in [8] that while the condition (2) alone is sufficient for the optimality in finite horizon, it is not the case for the infinite one, and gave beside an additional technical assumption which guarantees the optimality of MRAPs.

In this paper we propose a new optimality condition of MRAPs which is necessary and sufficient. Our approach is based on a characterization of the value function in terms of viscosity solutions [9] of a particular Hamilton-Jacobi equation. More precisely, we first prove that the value function of an equivalent problem (reasons for considering another problem will be given later) is the unique viscosity solution of a particular Hamilton-Jacobi equation. Then, we derive conditions under which the value of the objective along a MRAP, considered as a function of the initial condition $x_{0}$, is solution of this Hamilton-Jacobi equation. We finally deduce the optimality of MRAPs. In this approach, we need to consider a mathematical framework that guarantees the Hamilton-Jacobi equation to possess an unique solution. But uniqueness of solutions of first order partial differential equations is known to be difficult, especially when dealing with infinite horizon problems, for which the value function has to be defined on the whole $\mathbb{R}$, with no boundary condition. And no uniqueness result is known in the literature for the class of $C^{1}$ functions. This means that even when the value function turns out to be $C^{1}$, we still have to consider a larger class of functions. For this problem, Lipschitz continuous functions appear to be a well suited class, for which the viscosity solutions machinery provides uniqueness results. Furthermore, when more than one solution $\bar{x}$ provides an optimal MRAP, the value function is no longer differentiable, thus the required use of generalized solutions of the Hamilton-Jacobi equation, such as viscosity solutions.

The paper is organized as follows. In Section 2 we give assumptions and basic definitions that will be needed in the paper. In the following section, an equivalent optimal control problem is formulated and justified. Then we establish that the value function of the latter is the unique viscosity solution of a particular HamiltonianJacobi equation. In Section 4 we derive from the preceding result a necessary and sufficient condition for MRAPs to be optimal. In the last section we exhibit an example in which different possible occurrences of turnpikes (one or several) are given. Our approach provides in particular a criterion for the choice of turnpikes that are in competition. We also obtain situations in which our approach gives the optimality of a single MRAP, whereas the Hartl and Feichtinger sufficient conditions result does not. 


\section{Statement of the problem, ASsumptions And Definitions}

Let us consider the following set:

$$
A d m_{x_{0}}=\left\{x(.):\left[0, \infty\left[\rightarrow \mathbb{R}, A C, x(0)=x_{0}, \dot{x}(t) \in[-1,+1] \text { a.e. }\right\}\right.\right.
$$

whose elements are called the admissible paths. We also consider the functional, when it converges, given by:

$$
J[x(.)]=\int_{0}^{\rightarrow \infty} \mathrm{e}^{-\delta t}[A(x(t))+B(x(t)) \dot{x}(t)] \mathrm{d} t
$$

and we are interested by the following optimal control problem:

$$
\max _{x(.) \in \operatorname{Adm}\left(x_{0}\right)} J[x(.)]
$$

We assume that

$\left(H_{1}\right): A($.$) is twice differentiable and B($.$) is differentiable.$

$\left(H_{2}\right)$ : There exists two real numbers $k>0$ and $\gamma<\delta$ such that for all $x$

$$
\max \left(|A(x)|,\left|A^{\prime}(x)+\delta B(x)\right|,\left|A^{\prime \prime}(x)+\delta B^{\prime}(x)\right|\right) \leq k \mathrm{e}^{\gamma|x|} .
$$

These growth assumptions are more general than the usual ones when we one deals with necessary conditions (Euler condition, Maximum Principle).

The Euler equation, in this setting, is given by the following algebraic equation:

$$
C(x):=A^{\prime}(x)+\delta B(x)=0 .
$$

We denote by $E$ the set of the solutions of (5):

$$
E:=\{x \text { s.t. } C(x)=0\} .
$$

When the set $E$ is non empty, we consider the stationary paths $\bar{x}($.$) defined by$

$$
\bar{x}(t)=\bar{x} \quad \forall t \geq 0
$$

where $\bar{x} \in E$, which are candidate optimal solutions to the problem (4) (indeed we easily obtain the convergence of the improper integral associated to $J[]$.$) .$

Given $\bar{x} \in E$ and any $x_{0} \in \mathbb{R}$, we introduce the most rapid approach path from $x_{0}$ to $\bar{x}$, denoted by $\operatorname{MRAP}\left(x_{0}, \bar{x}\right)$ and defined as follows

$$
\begin{aligned}
& \text { if } x_{0} \geq \bar{x}, \quad \operatorname{MRAP}\left(x_{0}, \bar{x}\right)(t)=\mid \begin{array}{lr}
x_{0}-t \text { if } t \leq x_{0}-\bar{x} \\
\bar{x} & \text { if } t>x_{0}-\bar{x}
\end{array} \\
& \text { if } x_{0} \leq \bar{x}, \quad \operatorname{MRAP}\left(x_{0}, \bar{x}\right)(t)=\mid \begin{array}{lr}
x_{0}+t \text { if } t \leq \bar{x}-x_{0} \\
\bar{x} & \text { if } t>\bar{x}-x_{0} .
\end{array}
\end{aligned}
$$

Such paths are clearly admissible; moreover we can easily establish the convergence of the improper integral $J[$.$] .$

Our goal is to obtain necessary and sufficient conditions for the optimality of the $\operatorname{MRAP}\left(x_{0}, \bar{x}\right)$ with the help of a value function approach. Therefore, we begin by expressing the value of the objective $J[$.$] along the$ path $\hat{x}():.=\operatorname{MRAP}\left(x_{0}, \bar{x}\right)($.$) :$ 
- when $x_{0} \geq \bar{x}$ :

$$
\begin{aligned}
J[\hat{x}(.)] & =\frac{A\left(x_{0}\right)}{\delta}+\int_{0}^{\infty} \mathrm{e}^{-\delta t}\left[A^{\prime}(\hat{x}(t))+\delta B(\hat{x}(t))\right] \dot{\hat{x}}(t) \mathrm{d} t \\
& =\frac{A\left(x_{0}\right)}{\delta}-\frac{1}{\delta} \int_{0}^{x_{0}-\bar{x}} \mathrm{e}^{-\delta t}\left[A^{\prime}\left(x_{0}-t\right)+\delta B\left(x_{0}-t\right)\right] \mathrm{d} t .
\end{aligned}
$$

With the new variable $\xi=x_{0}-t$, it can be rewritten

$$
J[\hat{x}(.)]=\frac{A\left(x_{0}\right)}{\delta}+\frac{1}{\delta} \int_{x_{0}}^{\bar{x}} \mathrm{e}^{-\delta\left(x_{0}-\xi\right)}\left[A^{\prime}(\xi)+\delta B(\xi)\right] \mathrm{d} \xi ;
$$

- when $x_{0} \leq \bar{x}$, we then obtain

$$
J[\hat{x}(.)]=\frac{A\left(x_{0}\right)}{\delta}+\frac{1}{\delta} \int_{0}^{\bar{x}-x_{0}} \mathrm{e}^{-\delta t}\left[A^{\prime}\left(x_{0}+t\right)+\delta B\left(x_{0}+t\right)\right] \mathrm{d} t
$$

and with $\xi=x_{0}+t$,

$$
J[\hat{x}(.)]=\frac{A\left(x_{0}\right)}{\delta}+\frac{1}{\delta} \int_{x_{0}}^{\bar{x}} \mathrm{e}^{-\delta\left(\xi-x_{0}\right)}\left[A^{\prime}(\xi)+\delta B(\xi)\right] \mathrm{d} \xi .
$$

So in both case, we can write

$$
J\left[M R A P\left(x_{0}, \bar{x}\right)(.)\right]=\frac{A\left(x_{0}\right)}{\delta}+\frac{S\left(x_{0}, \bar{x}\right)}{\delta}
$$

where

$$
S\left(x_{0}, \bar{x}\right)=\int_{x_{0}}^{\bar{x}}\left(A^{\prime}(y)+\delta B(y)\right) \mathrm{e}^{-\delta\left|x_{0}-y\right|} \mathrm{d} y=\int_{x_{0}}^{\bar{x}} \mathrm{e}^{-\delta\left|x_{0}-y\right|} C(y) \mathrm{d} y .
$$

We underline that the function $S(.,$.$) , that will play a fundamental role in the sequel, depends only on the$ function $C($.$) , provided by the Euler equation (5).$

Now, to make the turnpike property more precise, we introduce the following definition: we call optimality basin of $\bar{x} \in E$ the set:

$$
\mathcal{B}(\bar{x}):=\left\{x_{0} \text { s.t. } \operatorname{MRAP}\left(x_{0}, \bar{x}\right) \text { is optimal }\right\} .
$$

$\bar{x}$ is then called a turnpike exactly when $\mathcal{B}(\bar{x})$ is not empty.

Finally, we introduce the value function $V($.$) , associated to the problem (4):$

$$
V\left(x_{0}\right)=\sup _{x(.) \in \operatorname{Adm}\left(x_{0}\right)} J[x(.)] .
$$

\section{A particular Hamilton-Jacobi equation}

As we recall in the introduction, the uniqueness of (generalized) solutions of first order partial differential equations defined on unbounded sets is known to be a delicate question. It can be obtained only for well chosen classes of functions (see for instance [1]). For this reason we do not characterize the value function $V($.$) itself, but$ a transformation of it, denoted by $Z($.$) in the sequel. The function Z($.$) is solution of another Hamilton-Jacobi$ equation, for which we are able to state a result of unique characterization in the class of BUC (bounded and uniformly continuous) functions. 
Proposition 3.1. Under Assumptions $\left(H_{1}\right)$ and $\left(H_{2}\right)$ the function $Z$ defined by

$$
Z(x)=\mathrm{e}^{-\eta \sqrt{x^{2}+1}}\left(V(x)-\frac{A(x)}{\delta}\right)
$$

where $\eta$ satisfies $\gamma<\eta<\delta$, is the unique bounded and uniformly continuous viscosity solution of the following Hamilton-Jacobi equation:

$$
\delta Z(x)-\left|Z^{\prime}(x)+\eta \frac{x}{\sqrt{x^{2}+1}} Z(x)+\frac{\mathrm{e}^{-\eta \sqrt{x^{2}+1}}}{\delta}\left(A^{\prime}(x)+\delta B(x)\right)\right|=0, \quad x \in \mathbb{R} .
$$

Proof. The proof is splitted into four steps.

Step 1. We derive a new equivalent problem.

Let fix $T \geq 0$. By an integration by parts we obtain:

$$
\begin{aligned}
\int_{0}^{T} \mathrm{e}^{-\delta t}[A(x(t))+B(x(t)) \dot{x}(t)] \mathrm{d} t & =\left[\frac{\mathrm{e}^{-\delta t} A(x(t))}{-\delta}\right]_{0}^{t}+\frac{1}{\delta} \int_{0}^{T} \mathrm{e}^{-\delta t}\left[A^{\prime}(x(t)) \dot{x}(t)+\delta B(x(t)) \dot{x}(t)\right] \mathrm{d} t \\
& =\frac{A\left(x_{0}\right)}{\delta}-\frac{A(x(T)) \mathrm{e}^{-\delta T}}{\delta}+\frac{1}{\delta} \int_{0}^{T} \mathrm{e}^{-\delta t}\left[A^{\prime}(x(t))+\delta B(x(t))\right] \dot{x}(t) \mathrm{d} t
\end{aligned}
$$

From the assumption $\left(H_{2}\right)$, we derive

$$
|A(x(T))| \mathrm{e}^{-\delta T} \leq k \mathrm{e}^{\gamma|x(T)|} \mathrm{e}^{-\delta T}
$$

and from $|\dot{x}(t)| \leq 1$, we have that for all $x(.) \in A d m_{x_{0}}$,

$$
|x(t)| \leq\left|x_{0}\right|+t
$$

therefore

$$
|A(x(T))| \mathrm{e}^{-\delta T} \leq k \mathrm{e}^{\gamma\left|x_{0}\right|} \mathrm{e}^{(\gamma-\delta) T} .
$$

Now as we assume that $\gamma-\delta<0$, we deduce

$$
A(x(T)) \mathrm{e}^{-\delta T} \longrightarrow 0 \text { when } T \longrightarrow+\infty .
$$

Then, the following limits (when they exist)

$$
\lim _{T \rightarrow \infty} \int_{0}^{T} \mathrm{e}^{-\delta t}[A(x(t))+B(x(t)) \dot{x}(t)] \mathrm{d} t, \lim _{T \rightarrow \infty} \int_{0}^{T} \mathrm{e}^{-\delta t}\left[A^{\prime}(x(t))+\delta B(x(t))\right] \dot{x}(t) \mathrm{d} t
$$

satisfy the equality

$$
\int_{0}^{\infty} \mathrm{e}^{-\delta t}[A(x(t))+B(x(t)) \dot{x}(t)] \mathrm{d} t=\frac{A\left(x_{0}\right)}{\delta}+\frac{1}{\delta} \int_{0}^{\infty} \mathrm{e}^{-\delta t}\left[A^{\prime}(x(t))+\delta B(x(t))\right] \dot{x}(t) \mathrm{d} t
$$

(more precisely, if one exists, then the other one also). 
Therefore our problem and the new following problem

$$
\max _{x(.) \in \operatorname{Adm}\left(x_{0}\right)} \int_{0}^{\infty} \mathrm{e}^{-\delta s}\left[A^{\prime}(x(s))+\delta B(x(s))\right] \dot{x}(s) \mathrm{d} s
$$

possess the same optimal solutions (if they exist). Then, we shall say that the optimization problems (4) and (10) are equivalent, when both of them admit a solution.

Now we prove that the improper integral in (10) converges always on the set $\operatorname{Adm}\left(x_{0}\right)$. Therefore the integral in (3) also converges on $\operatorname{Adm}\left(x_{0}\right)$ and the two problems are equivalent.

From $|\dot{x}(t)| \leq 1$, we have

$$
\left|\int_{0}^{T} \mathrm{e}^{-\delta s}\left[A^{\prime}(x(s))+\delta B(x(s))\right] \dot{x}(s) \mathrm{d} s\right| \leq \int_{0}^{T} \mathrm{e}^{-\delta t}\left|A^{\prime}(x(t))+\delta B(x(t))\right| \mathrm{d} t,
$$

and with the assumption $\left(H_{2}\right)$, we can deduce that

$$
\begin{aligned}
\int_{0}^{T} \mathrm{e}^{-\delta s}\left[A^{\prime}(x(s))+\delta B(x(s))\right] \dot{x}(s) \mathrm{d} s & \leq k \int_{0}^{T} \mathrm{e}^{-\delta t} \mathrm{e}^{\gamma|x(t)|} \mathrm{d} t \\
& \leq k \int_{0}^{T} \mathrm{e}^{-\delta t} \mathrm{e}^{\gamma\left(\left|x_{0}\right|+t\right)} \mathrm{d} t=k \mathrm{e}^{\gamma\left|x_{0}\right|} \int_{0}^{T} \mathrm{e}^{(\gamma-\delta) t} \\
& =k \mathrm{e}^{\gamma\left|x_{0}\right|}\left[\frac{\mathrm{e}^{(\gamma-\delta) t}}{\gamma-\delta}\right]_{0}^{T}=k \mathrm{e}^{\gamma\left|x_{0}\right|}\left(\frac{\mathrm{e}^{(\gamma-\delta) T}}{\gamma-\delta}-1\right) .
\end{aligned}
$$

We have assumed that $\gamma-\delta<0$, and therefore the improper integral of the new problem is an absolutely convergent integral, then a convergent integral.

Denote $W($.$) the value function of the new problem (10). We deduce moreover:$

i)

$$
\int_{0}^{\infty}\left|\mathrm{e}^{-\delta t}\left[A^{\prime}(x(t))+\delta B(x(t))\right] \dot{x}(t)\right| \mathrm{d} t \leq \frac{k \mathrm{e}^{\gamma\left|x_{0}\right|}}{\gamma-\delta}
$$

ii) for the particular path $\widetilde{x}($.$) such that \dot{\widetilde{x}}()=$.0 which belongs to $A d m\left(x_{0}\right)$, we have

$$
\int_{0}^{\infty} \mathrm{e}^{-\delta s}\left[A^{\prime}(\widetilde{x}(s))+\delta B(\widetilde{x}(s))\right] \dot{\widetilde{x}}(s) \mathrm{d} s=0
$$

and therefore

We then have established that:

$$
W\left(x_{0}\right) \geq 0, \quad \forall x_{0} \in \mathbb{R}
$$

$$
V\left(x_{0}\right)-\frac{A\left(x_{0}\right)}{\delta}=\frac{W\left(x_{0}\right)}{\delta} \geq 0, \quad \forall x_{0} .
$$

The function $W($.$) doesn't belong to the class of the BUC functions. Therefore we introduce the function Z($. defined by

$$
\forall x, \quad Z(x)=\mathrm{e}^{-\eta \sqrt{x^{2}+1}} \frac{W(x)}{\delta}=\mathrm{e}^{-\eta \sqrt{x^{2}+1}}\left(V(x)-\frac{A(x)}{\delta}\right)
$$

where $\eta$ satisfies $\gamma<\eta<\delta$.

Step 2. The function $Z($.) is BUC (bounded and uniformly continuous). 
In order to simplify notations we posit

$$
\varphi(x)=\sqrt{x^{2}+1}
$$

and we define the following function $\Gamma$ given by

$$
\Gamma_{t}(x)=\mathrm{e}^{-\eta t} \mathrm{e}^{-\eta \varphi(x)} C(x+\psi(t)), \quad \forall(t, x) .
$$

From the definition of the value function of problem (10), for each $\epsilon>0$ there exists a path $x_{\epsilon} \in A d m_{x_{0}}$ such that we have

$$
\int_{0}^{\infty} \mathrm{e}^{-\delta t} C\left(x_{\epsilon}(t)\right) \dot{x}_{\epsilon}(t) \mathrm{d} t>W\left(x_{0}\right)-\epsilon
$$

then we obtain the two following inequalities

$$
\begin{gathered}
-\frac{W\left(x_{0}\right)}{\delta}=-\left(V\left(x_{0}\right)-\frac{A\left(x_{0}\right)}{\delta}\right)>-\frac{1}{\delta} \int_{0}^{\infty} \mathrm{e}^{-\delta t} C\left(x_{\epsilon}(t)\right) \dot{x}_{\epsilon}(t) \mathrm{d} t-\frac{\epsilon}{\delta}, \\
-Z\left(x_{0}\right)=-\left(V\left(x_{0}\right)-\frac{A\left(x_{0}\right)}{\delta}\right) \mathrm{e}^{-\eta \varphi\left(x_{0}\right)}>-\frac{1}{\delta} \int_{0}^{\infty} \mathrm{e}^{-\delta t} \mathrm{e}^{-\eta \varphi\left(x_{0}\right)} C\left(x_{\epsilon}(t)\right) \dot{x}_{\epsilon}(t) \mathrm{d} t-\frac{\epsilon}{\delta} .
\end{gathered}
$$

Now, $x_{\epsilon}($.$) being an absolutely continuous function, it satisfies therefore$

$$
x_{\epsilon}(t)=x_{0}+\int_{0}^{t} \dot{x}_{\epsilon}(s) \mathrm{d} s:=x_{0}+\psi(t)
$$

and the path $\bar{x}_{\epsilon}($.$) defined by \bar{x}_{\epsilon}(t)=\bar{x}_{0}+\psi(t)$ belongs to $A d m_{\bar{x}_{0}}$. This path is sub-optimal for the problem (10), and we deduce that

$$
V\left(\bar{x}_{0}\right)-\frac{A\left(\bar{x}_{0}\right)}{\delta}=\frac{W\left(\bar{x}_{0}\right)}{\delta} \geq \frac{1}{\delta} \int_{0}^{\infty} \mathrm{e}^{-\delta t} C\left(\bar{x}_{\epsilon}(t)\right) \dot{\bar{x}}_{\epsilon}(t) \mathrm{d} t
$$

then

$$
Z\left(\bar{x}_{0}\right)=\left(V\left(\bar{x}_{0}\right)-\frac{A\left(\bar{x}_{0}\right)}{\delta}\right) \mathrm{e}^{-\eta \varphi\left(\bar{x}_{0}\right)} \geq \frac{1}{\delta} \int_{0}^{\infty} \mathrm{e}^{-\delta t} \mathrm{e}^{-\eta \varphi\left(\bar{x}_{0}\right)} C\left(\bar{x}_{\epsilon}(t)\right) \dot{\bar{x}}_{\epsilon}(t) \mathrm{d} t .
$$

Finally, by using the fact that $\dot{\bar{x}}_{\epsilon}(t)=\dot{x}_{\epsilon}(t)=\dot{\psi}(t)$ a.e., we can compute the difference:

$$
\begin{aligned}
Z\left(\bar{x}_{0}\right)-Z\left(x_{0}\right) & \geq \frac{1}{\delta} \int_{0}^{\infty} \mathrm{e}^{-\delta t} \mathrm{e}^{-\eta \varphi\left(\bar{x}_{0}\right)} C\left(\bar{x}_{\epsilon}(t)\right) \dot{x}_{\epsilon}(t) \mathrm{d} t-\frac{1}{\delta} \int_{0}^{\infty} \mathrm{e}^{-\delta t} \mathrm{e}^{-\eta \varphi\left(x_{0}\right)} C\left(x_{\epsilon}(t)\right) \dot{x}_{\epsilon}(t) \mathrm{d} t-\frac{\epsilon}{\delta} \\
& =\frac{1}{\delta} \int_{0}^{\infty} \mathrm{e}^{-\delta t}\left[\mathrm{e}^{-\eta \varphi\left(\bar{x}_{0}\right)} C\left(\bar{x}_{\epsilon}(t)\right)-\mathrm{e}^{-\eta \varphi\left(x_{0}\right)} C\left(x_{\epsilon}(t)\right)\right] \dot{\psi}(t) \mathrm{d} t-\frac{\epsilon}{\delta}
\end{aligned}
$$

From $\dot{\psi}(t) \geq-1$, which is valid for a.e. $t$, we obtain

$$
\begin{aligned}
Z\left(\bar{x}_{0}\right)-Z\left(x_{0}\right) & \geq-\frac{1}{\delta} \int_{0}^{\infty} \mathrm{e}^{-\delta t}\left|\mathrm{e}^{-\eta \varphi\left(\bar{x}_{0}\right)} C\left(\bar{x}_{\epsilon}(t)\right)-\mathrm{e}^{-\eta \varphi\left(x_{0}\right)} C\left(x_{\epsilon}(t)\right)\right| \mathrm{d} t-\frac{\epsilon}{\delta} \\
& =-\frac{1}{\delta} \int_{0}^{\infty} \mathrm{e}^{(\eta-\delta) t}\left|\mathrm{e}^{-\eta t} \mathrm{e}^{-\eta \varphi\left(\bar{x}_{0}\right)} C\left(\bar{x}_{0}+\psi(t)\right)-\mathrm{e}^{-\eta t} \mathrm{e}^{-\eta \varphi\left(x_{0}\right)} C\left(x_{0}+\psi(t)\right)\right| \mathrm{d} t-\frac{\epsilon}{\delta}
\end{aligned}
$$

Finally in terms of the function $\Gamma$, we have established that

$$
Z\left(\bar{x}_{0}\right)-Z\left(x_{0}\right) \geq-\frac{1}{\delta} \int_{0}^{\infty} \mathrm{e}^{(\eta-\delta) t}\left|\Gamma_{t}\left(\bar{x}_{0}\right)-\Gamma_{t}\left(x_{0}\right)\right| \mathrm{d} t-\frac{\epsilon}{\delta} .
$$


In order to obtain an estimate for the right hand side of the inequality (13), we evaluate the derivative of $\Gamma$ w.r.t. $x$. First we observe the two following facts:

i) $[\varphi(x+\psi(t))]^{2}=\left[(x+\psi(t))^{2}+1\right]^{2}=x^{2}+1+\psi^{2}(t)+2 x \psi(t)=\varphi^{2}(x)+\psi^{2}(t)+2 x \psi(t)$.

But we know that $|\psi(t)| \leq t$ and that $|x| \leq \varphi(x)$, therefore

$$
\begin{aligned}
{[\varphi(x+\psi(t))]^{2} } & \leq \varphi^{2}(x)+\psi^{2}(t)+2|x| \cdot|\psi(t)| \\
& \leq \varphi^{2}(x)+\psi^{2}(t)+2 \varphi(x) t \\
& \leq \varphi^{2}(x)+t^{2}+2 \varphi(x) t=[\varphi(x)+t]^{2} .
\end{aligned}
$$

We can then deduce

ii)

$$
\varphi(x+\psi(t)) \leq \varphi(x)+t
$$

From these two remarks, we obtain

$$
|x+\psi(t)| \leq|x|+|\psi(t)| \leq \varphi(x)+t .
$$

$$
\begin{aligned}
\left|\frac{\mathrm{d} \Gamma_{t}(x)}{\mathrm{d} x}\right| & =\left|\mathrm{e}^{\eta t} \mathrm{e}^{-\eta \varphi(x)}\left[C^{\prime}(x+\psi(t))-\eta \varphi^{\prime}(x) C(x+\psi(t))\right]\right| \\
& =\mathrm{e}^{-\eta(t+\psi(x))}\left|C^{\prime}(x+\psi(t))-\eta \varphi^{\prime}(x) C(x+\psi(t))\right| \\
& \leq \mathrm{e}^{-\eta(x+\psi(t))}\left|C^{\prime}(x+\psi(t))\right|+\mathrm{e}^{-\eta(x+\psi(t))} \eta\left|\varphi^{\prime}(x)\right| .|C(x+\psi(t))| .
\end{aligned}
$$

But $\varphi^{\prime}(x)=|x| / \sqrt{x^{2}+1} \leq 1$, and with $\left(H_{2}\right)$ we obtain

$$
\left|\frac{\mathrm{d} \Gamma_{t}(x)}{\mathrm{d} x}\right| \leq \mathrm{e}^{-\eta \varphi(x+\psi(t))} k \mathrm{e}^{\gamma|x+\psi(t)|}+\eta \mathrm{e}^{-\eta \varphi(x+\psi(t))} k \mathrm{e}^{\gamma|x+\psi(t)|} .
$$

Therefore from (14) and (15), we have

$$
\left|\frac{\mathrm{d} \Gamma_{t}(x)}{\mathrm{d} x}\right| \leq(1+\eta) k \mathrm{e}^{(\gamma-\eta)(\varphi(x)+t)} .
$$

Let us recall that by assumption that $\eta>\gamma$, and that $\varphi(x)=\sqrt{x^{2}+1} \geq 0$, we finally obtain the following bound

$$
\left|\frac{\mathrm{d} \Gamma_{t}(x)}{\mathrm{d} x}\right| \leq K:=(1+\eta) k \text {. }
$$

Hence the derivative of $\Gamma$ is uniformly bounded for all $t$ and $x$.

We are now able to prove the main result of this step: the function $Z($.$) is BUC.$

By the use of the mean-value theorem, we deduce that

$$
\exists x \text { s. t. }\left|\Gamma_{t}\left(\bar{x}_{0}\right)-\Gamma_{t}\left(x_{0}\right)\right|=\left|\bar{x}_{0}-x_{0}\right| \cdot\left|\frac{\mathrm{d} \Gamma_{t}(x)}{\mathrm{d} x}\right| \leq K\left|\bar{x}_{0}-x_{0}\right|
$$

and therefore, using the assumption that $\eta-\delta<0$, we obtain that

$$
\begin{aligned}
Z\left(\bar{x}_{0}\right)-Z\left(x_{0}\right) & \geq-\frac{1}{\delta} \int_{0}^{\infty} \mathrm{e}^{(\eta-\delta) t} K\left|\bar{x}_{0}-x_{0}\right| \mathrm{d} t-\frac{\epsilon}{\delta} \\
& =-\frac{K}{\delta}\left|\bar{x}_{0}-x_{0}\right|\left[\frac{\mathrm{e}^{(\eta-\delta) t}}{\eta-\delta}\right]_{0}^{\infty}-\frac{\epsilon}{\delta}=-\frac{K}{\delta(\delta-\eta)}\left|\bar{x}_{0}-x_{0}\right|-\frac{\epsilon}{\delta}
\end{aligned}
$$


This inequality holds for any $\epsilon>0$, and then we have

$$
Z\left(\bar{x}_{0}\right)-Z\left(x_{0}\right) \geq-\frac{K}{\delta(\delta-\eta)}\left|\bar{x}_{0}-x_{0}\right| .
$$

Exchanging $\bar{x}_{0}$ and $x_{0}$, we finally obtain

$$
\left|Z\left(\bar{x}_{0}\right)-Z\left(x_{0}\right)\right| \leq \frac{K}{\delta(\delta-\eta)}\left|\bar{x}_{0}-x_{0}\right| .
$$

On the other hand, from (9) and (11), we deduce that for any $x(.) \in A d m_{x_{0}}$.

$$
\begin{aligned}
\left|\int_{0}^{\infty} \mathrm{e}^{-\delta t}[A(x(t))+B(x(t)) \dot{x}(t)] \mathrm{d} t-\frac{A\left(x_{0}\right)}{\delta}\right| & \leq \frac{1}{\delta} \int_{0}^{\infty} \mathrm{e}^{-\delta t}\left|\left[A^{\prime}(x(t))+\delta B(x(t))\right] \dot{x}(t)\right| \mathrm{d} t \\
& \leq \frac{k}{\delta(\delta-\gamma)} \mathrm{e}^{\gamma\left|x_{0}\right|} .
\end{aligned}
$$

Therefore

and

$$
\left|V\left(x_{0}\right)-\frac{A\left(x_{0}\right)}{\delta}\right| \leq \frac{k}{\delta(\delta-\gamma)} \mathrm{e}^{\gamma\left|x_{0}\right|}
$$

$$
|Z(x)|=\left|\mathrm{e}^{-\eta \varphi(x)}\left(V(x)-\frac{A(x)}{\delta}\right)\right| \leq \frac{k}{\delta(\delta-\gamma)} \mathrm{e}^{-\eta \varphi(x)} \mathrm{e}^{\gamma|x|}
$$

But we have $\varphi(x) \geq|x|$, and therefore

$$
|Z(x)| \leq \frac{k}{\delta(\delta-\gamma)} \mathrm{e}^{(\gamma-\eta)|x|}
$$

We then deduce that $Z(x) \rightarrow 0$ when $|x| \rightarrow+\infty$, by the assumption that $\eta>\gamma$. Being Lipschitz, $Z($.$) is$ continuous and it follows that it is bounded.

We then conclude that $Z($.$) , being Lipschitz continuous and bounded, belongs to the class of BUC functions.$

Step 3. The function $Z($.$) as a viscosity solution of (8).$

From the dynamic programming equation applied to the value function $V($.$) , we obtain, for all T \geq 0$, that

$$
\begin{aligned}
V\left(x_{0}\right) & =\sup _{x(.) \in A d m_{x_{0}}}\left\{\int_{0}^{T} \mathrm{e}^{-\delta t}(A(x(t))+B(x(t))) \dot{x}(t) \mathrm{d} t+\mathrm{e}^{-\delta T} V(x(T))\right\} \\
& =\sup _{x(.) \in A d m_{x_{0}}}\left\{\left[\frac{\mathrm{e}^{-\delta t}}{\delta} A(x(t))\right]_{T}^{0}+\frac{1}{\delta} \int_{0}^{T} \mathrm{e}^{-\delta t}\left[A^{\prime}(x(t))+\delta B(x(t))\right] \dot{x}(t) \mathrm{d} t+\mathrm{e}^{-\delta T} V(x(T))\right\} \\
& =\frac{A\left(x_{0}\right)}{\delta}+\sup _{x(.) \in A d m_{x_{0}}}\left\{\frac{1}{\delta} \int_{0}^{T} \mathrm{e}^{-\delta t}\left[A^{\prime}(x(t))+\delta B(x(t))\right] \dot{x}(t) \mathrm{d} t+\mathrm{e}^{-\delta T}\left(V(x(T))-\frac{A(x(T))}{\delta}\right)\right\}
\end{aligned}
$$

From $Z(x) \mathrm{e}^{\eta \varphi(x)}=V(x)-A(x) / \delta$, we derive that

$$
Z\left(x_{0}\right) \mathrm{e}^{\eta \varphi\left(x_{0}\right)}=\sup _{A d m_{x_{0}}}\left\{\frac{1}{\delta} \int_{0}^{T} \mathrm{e}^{-\delta t}\left[A^{\prime}(x(t))+\delta B(x(t))\right] \dot{x}(t) \mathrm{d} t+\mathrm{e}^{-\delta T} Z(x(T)) \mathrm{e}^{\eta \varphi(x(T))}\right\} .
$$


From this last property we derive the main result of this step that establishes that the function $Z($.$) is a viscosity$ solution of the Hamilton-Jacobi equation (8).

Let $\Phi$ be a test function, i.e. a $C^{1}$ function which admits $x_{0}$ as a (local) maximum. Without any loss of generality, we can also suppose that $Z\left(x_{0}\right)-\Phi\left(x_{0}\right)=0$.

Therefore, at least locally, we have

$$
\Phi(x) \geq Z(x)
$$

and then

$$
\begin{aligned}
\mathrm{e}^{\eta \varphi\left(x_{0}\right)} \Phi\left(x_{0}\right) & =\mathrm{e}^{\eta \varphi\left(x_{0}\right)} Z\left(x_{0}\right) \\
& =\sup _{A d m_{x_{0}}}\left\{\frac{1}{\delta} \int_{0}^{T} \mathrm{e}^{-\delta t}\left[A^{\prime}(x(t))+\delta B(x(t))\right] \dot{x}(t) \mathrm{d} t+\mathrm{e}^{-\delta T} Z(x(T)) \mathrm{e}^{\eta \varphi(x(T))}\right\} \\
& \leq \sup _{A d m_{x_{0}}}\left\{\frac{1}{\delta} \int_{0}^{T} \mathrm{e}^{-\delta t}\left[A^{\prime}(x(t))+\delta B(x(t))\right] \dot{x}(t) \mathrm{d} t+\mathrm{e}^{-\delta T} \Phi(x(T)) \mathrm{e}^{\eta \varphi(x(T))}\right\} .
\end{aligned}
$$

From this inequality, we can derive that

$$
\sup _{A d m_{x_{0}}}\left\{\frac{1}{\delta} \int_{0}^{T} \mathrm{e}^{-\delta t}\left[A^{\prime}(x(t))+\delta B(x(t))\right] \dot{x}(t) \mathrm{d} t+\mathrm{e}^{-\delta T} \Phi(x(T)) \mathrm{e}^{\eta \varphi(x(T))}\right\}-\mathrm{e}^{\eta \varphi\left(x_{0}\right)} \Phi\left(x_{0}\right) \geq 0
$$

Now we remark that

$$
\frac{\mathrm{d}}{\mathrm{d} t}\left[\mathrm{e}^{-\delta t+\eta \varphi(x(t))} \Phi(x(t))\right]=\mathrm{e}^{-\delta t} \mathrm{e}^{\eta \varphi(x(t))}\left[-\delta \Phi(x(t))+\left(\eta \varphi^{\prime}(x(t)) \Phi(x(t))+\Phi^{\prime}(x(t))\right) \dot{x}(t)\right],
$$

and obtain

$$
\int_{0}^{t} \mathrm{e}^{\eta \varphi(x(s))}\left[\left(\eta \varphi^{\prime}(x(s)) \Phi(x(s))+\Phi^{\prime}(x(s))\right) \dot{x}(s)-\delta \Phi(x(s))\right] \mathrm{e}^{-\delta s} \mathrm{~d} s=\mathrm{e}^{-\delta t} \mathrm{e}^{\eta \varphi(x(t))} \Phi(x(t))-\mathrm{e}^{\eta \varphi\left(x_{0}\right)} \Phi\left(x_{0}\right) .
$$

The inequality (17) becomes now

$$
\begin{array}{r}
\sup _{\operatorname{Adm}\left(x_{0}\right)}\left[\int _ { 0 } ^ { t } \left\{\frac{1}{\delta}\left[A^{\prime}(x(s))+\delta B(x(s))\right] \dot{x}(s) \mathrm{e}^{-\eta \varphi(x(s))}+\left(\eta \varphi^{\prime}(x(s)) \Phi(x(s))\right.\right.\right. \\
\left.\left.+\Phi^{\prime}(x(s))\right) \dot{x}(s)-\delta \Phi(x(s))\right\} \mathrm{e}^{\left.\eta \varphi(x(s)) \mathrm{e}^{-\delta s} \mathrm{~d} s\right] \geq 0}
\end{array}
$$

and therefore

$$
\begin{aligned}
& \sup _{\operatorname{Adm}\left(x_{0}\right)}\left[\frac { 1 } { t } \int _ { 0 } ^ { t } \left\{\left(\frac{1}{\delta}\left[A^{\prime}(x(s))+\delta B(x(s))\right] \mathrm{e}^{-\eta \varphi(x(s))}+\eta \varphi^{\prime}(x(s)) \Phi(x(s))+\Phi^{\prime}(x(s))\right) \dot{x}(s)\right.\right. \\
& \left.-\delta \Phi(x(s))\} \mathrm{e}^{\eta \varphi(x(s)) \mathrm{e}^{-\delta s}} \mathrm{~d} s\right] \geq 0
\end{aligned}
$$

Hence for the paths $x(.) \in A d m\left(x_{0}\right)$ such that $\dot{x}($.$) is continuous at 0$, we obtain when $t$ goes to 0 :

$$
\sup _{\dot{x}_{0} \in[-1,+1]}\left[\left\{\left(\frac{1}{\delta}\left(A^{\prime}\left(x_{0}\right)+\delta B\left(x_{0}\right)\right) \mathrm{e}^{-\eta \varphi\left(x_{0}\right)}+\eta \varphi^{\prime}\left(x_{0}\right) \Phi\left(x_{0}\right)+\Phi^{\prime}\left(x_{0}\right)\right) \dot{x}_{0}-\delta \Phi\left(x_{0}\right)\right\} \mathrm{e}^{\eta \varphi\left(x_{0}\right)}\right] \geq 0
$$


from which we derive that $V$ is a viscosity sub-solution of $(8)$ at $x_{0}$, for any $x_{0}$, i.e.

$$
\delta \Phi(x)-\left|\frac{1}{\delta}\left(A^{\prime}(x)+\delta B(x)\right) \mathrm{e}^{-\eta \varphi(x)}+\eta \varphi^{\prime}(x) \Phi(x)+\Phi^{\prime}(x)\right| \leq 0 .
$$

We now consider test functions $\Phi($.$) such that Z()-.\Phi($.$) admits x_{0}$ as a local minimum. We can also suppose that $Z\left(x_{0}\right)-\Phi\left(x_{0}\right)=0$ and then, locally

From (16), we derive that

$$
Z(x)-\Phi(x) \geq 0
$$

$$
\Phi\left(x_{0}\right) \mathrm{e}^{\eta \varphi\left(x_{0}\right)}=Z\left(x_{0}\right) \mathrm{e}^{\eta \varphi\left(x_{0}\right)} \geq \frac{1}{\delta} \int_{0}^{t}\left[A^{\prime}(x(s))+\delta B(x(s))\right] \dot{x}(s) \mathrm{e}^{-\delta s} \mathrm{~d} s+\mathrm{e}^{-\delta t} Z(x(t)) \mathrm{e}^{\eta \varphi(x(t))}
$$

where $x($.$) stands for any path in \operatorname{Adm}\left(x_{0}\right)$. We then deduce that

$$
\Phi\left(x_{0}\right) \mathrm{e}^{\eta \varphi\left(x_{0}\right)} \geq \frac{1}{\delta} \int_{0}^{t}\left[A^{\prime}(x(s))+\delta B(x(s))\right] \dot{x}(s) \mathrm{e}^{-\delta s} \mathrm{~d} s+\Phi(x(t)) \mathrm{e}^{\eta \varphi(x(t))} \mathrm{e}^{-\delta t}
$$

and we obtain

$$
\frac{1}{t}\left[\Phi(x(t)) \mathrm{e}^{\eta \varphi(x(t))} \mathrm{e}^{-\delta t}-\Phi\left(x_{0}\right) \mathrm{e}^{\eta \varphi\left(x_{0}\right)}\right] \leq-\frac{1}{t} \int_{0}^{t} \frac{1}{\delta}\left[A^{\prime}(x(s))+\delta B(x(s))\right] \dot{x}(s) \mathrm{e}^{-\delta s} \mathrm{~d} s .
$$

For the paths $x($.$) such that \dot{x}($.$) is continuous at 0$ we have

$$
\frac{\mathrm{d}}{\mathrm{d} t}\left[\Phi(x(t)) \mathrm{e}^{\eta \varphi(x(t))-\delta t}\right]_{t=0} \leq-\frac{1}{\delta}\left[A^{\prime}\left(x_{0}\right)+\delta B\left(x_{0}\right)\right] \dot{x}(0)
$$

which is equivalent to

$$
\Phi^{\prime}\left(x_{0}\right) \dot{x}(0) \mathrm{e}^{\eta \varphi\left(x_{0}\right)}+\Phi\left(x_{0}\right)\left(\eta \varphi^{\prime}\left(x_{0}\right) \dot{x}(0)-\delta\right) \mathrm{e}^{\eta \varphi\left(x_{0}\right)} \leq-\frac{1}{\delta}\left[A^{\prime}\left(x_{0}\right)+\delta B\left(x_{0}\right)\right] \dot{x}(0)
$$

and therefore

$$
\left[\Phi^{\prime}\left(x_{0}\right)+\eta \varphi^{\prime}\left(x_{0}\right) \Phi\left(x_{0}\right)+\frac{1}{\delta}\left[A^{\prime}\left(x_{0}\right)+\delta B\left(x_{0}\right)\right] \mathrm{e}^{-\eta \varphi\left(x_{0}\right)}\right] \dot{x}(0)-\delta \Phi\left(x_{0}\right) \leq 0 .
$$

This inequality is satisfied by any $\dot{x}(0) \in[-1,1]$ and we can then conclude that

$$
\delta \Phi(x)-\left|\frac{1}{\delta}\left(A^{\prime}(x)+\delta B(x)\right) \mathrm{e}^{-\eta \varphi(x)}+\eta \varphi^{\prime}(x) \Phi(x)+\Phi^{\prime}(x)\right| \geq 0 .
$$

We have finally proved that $Z($.$) is a viscosity solution of (8).$

Step 4. Uniqueness of BUC solutions of the Hamilton-Jacobi equation (8).

We prove that uniqueness results for BUC solutions of first order p.d.e. apply (see for instance Th. 2.11 in $[1])$. Let us denote by $H$ the Hamiltonian defined by

$$
H(x, z, p)=\delta z-\left|\frac{1}{\delta}\left(A^{\prime}(x)+\delta B(x)\right) \mathrm{e}^{-\eta \varphi(x)}+\eta \varphi^{\prime}(x) z+p\right|,
$$

which corresponds to the left member of (8). We prove now that $H$ satisfies the three following properties: 
i) Let $z_{1} \leq z_{2}$, we have

$$
\begin{aligned}
& H\left(x, z_{2}, p\right)-H\left(x, z_{1}, p\right)=\delta\left(z_{2}-z_{1}\right)+\left|\frac{1}{\delta}\left(A^{\prime}(x)+\delta B(x)\right) \mathrm{e}^{-\eta \varphi(x)}+\eta \varphi^{\prime}(x) z_{1}+p\right| \\
& -\left|\frac{1}{\delta}\left(A^{\prime}(x)+\delta B(x)\right) \mathrm{e}^{-\eta \varphi(x)}+\eta \varphi^{\prime}(x) z_{2}+p\right| \\
& \geq-\left|\eta \varphi^{\prime}(x)\left(z_{2}-z_{1}\right)\right|+\delta\left(z_{2}-z_{1}\right) \\
& =\left(\delta-\eta \varphi^{\prime}(x)\right)\left(z_{2}-z_{1}\right) \geq(\delta-\eta)\left(z_{2}-z_{1}\right)
\end{aligned}
$$

because $\delta-\eta>0$, which is the first assumption in [1];

ii) We also have

$$
\begin{aligned}
\left|H\left(x_{1}, z, p\right)-H\left(x_{2}, z, p\right)\right|=|-| p+\eta \varphi^{\prime}\left(x_{1}\right) z+\frac{1}{\delta}\left(A^{\prime}\left(x_{1}\right)+\delta B\left(x_{1}\right)\right) \mathrm{e}^{-\eta \varphi\left(x_{1}\right)} \mid-\delta z \\
\quad+\left|p+\eta \varphi^{\prime}\left(x_{2}\right) z+\frac{1}{\delta}\left(A^{\prime}\left(x_{2}\right)+\delta B\left(x_{2}\right)\right) \mathrm{e}^{-\eta \varphi\left(x_{2}\right)}\right|+\delta z \mid \\
\leq \mid \eta z\left(\varphi^{\prime}\left(x_{2}\right)-\varphi^{\prime}\left(x_{1}\right)\right)+\frac{1}{\delta}\left[\left(A^{\prime}\left(x_{2}\right)+\delta B\left(x_{2}\right)\right) \mathrm{e}^{-\eta \varphi\left(x_{2}\right)}\right. \\
\left.\quad-\left(A^{\prime}\left(x_{1}\right)+\delta B\left(x_{1}\right)\right) \mathrm{e}^{-\eta \varphi\left(x_{1}\right)}\right] \mid \\
\leq \eta|z|\left|\left(\varphi^{\prime}\left(x_{2}\right)-\varphi^{\prime}\left(x_{1}\right)\right)+\frac{1}{\delta}\right|\left(A^{\prime}\left(x_{2}\right)+\delta B\left(x_{2}\right)\right) \mathrm{e}^{-\eta \varphi\left(x_{2}\right)} \\
\quad-\left(A^{\prime}\left(x_{1}\right)+\delta B\left(x_{1}\right)\right) \mathrm{e}^{-\eta \varphi\left(x_{1}\right)} \mid \\
\leq\left(m_{1, R}+m_{2, R}\right)\left(\left|x_{1}-x_{2}\right|(1+|p|)\right)
\end{aligned}
$$

for $|z| \leq R$, with $m_{i, R}(t) \rightarrow 0, t \rightarrow 0$, which is the second assumption in [1];

iii) We suppose that $z \in[-R, R]$ and $p \in \bar{B}_{R}$ (the compact $R$-disk), then $H$ is uniformly continuous on $\mathbb{R} \times[-R, R] \times \mathbb{R}$, because $x \mapsto \varphi^{\prime}(x)$ and $x \mapsto\left(A^{\prime}(x)+\delta B(x)\right) \mathrm{e}^{-\eta \varphi(x)}$ are.

Therefore we have proved the announced proposition.

\section{TURnPike OPTIMAlity}

Given the set $E$ of solutions of the Euler equation (5), assumed to be non empty and finite, we define the following function $\bar{S}$, which is playing an important role in the following:

$$
\bar{S}(x):=\max _{\bar{x} \in E} S(x, \bar{x}) .
$$

Proposition 4.1. Let us assume $\left(H_{1}\right)-\left(H_{2}\right)$, and suppose that $E$ is non empty and finite. Then the following statements are equivalent:

i) For any $x_{0}$, there exists a turnpike $\bar{x} \in E$ (i.e. there exists $\bar{x}$ such that $x_{0} \in \mathcal{B}(\bar{x})$ );

ii) We have

$$
\bar{S}(x) \geq 0, \quad \forall x \in \mathbb{R} .
$$

Moreover the value function of the problem is given by

$$
V(x)=(A(x)+\bar{S}(x)) / \delta .
$$


Proof. Assume that the set $E$ has a finite cardinality, and that (20) is fulfilled.

Let $x_{0}$ be an initial condition and $\bar{x} \in \arg \max _{\bar{\xi} \in E} S\left(x_{0}, \bar{\xi}\right)$.

Then using (6), we can write

$$
J\left[M R A P\left(x_{0}, \bar{x}\right)\right]=\frac{A\left(x_{0}\right)}{\delta}+\frac{S\left(x_{0}, \bar{x}\right)}{\delta}=\frac{A\left(x_{0}\right)}{\delta}+\frac{\bar{S}\left(x_{0}\right)}{\delta} .
$$

Therefore the $\operatorname{MRAP}\left(x_{0}, \bar{x}\right)$ are optimal paths for any $x_{0}$ if and only if the function

$$
x \mapsto \frac{A(x)}{\delta}+\frac{\bar{S}(x)}{\delta}
$$

is the value function that we have characterized in our preceding proposition, that is to say

$$
Z(x)=\mathrm{e}^{-\eta \phi(x)} \frac{\bar{S}(x)}{\delta} .
$$

The proof of this result is then given in two steps:

Step 1. The function $Z($.$) is BUC.$

Let $x \in \mathbb{R}$, we have for a particular $\bar{x} \in E$ :

$$
Z(x)=\frac{1}{\delta} \int_{x}^{\bar{x}} \mathrm{e}^{-\delta|x-\xi|} \mathrm{e}^{-\eta \varphi(x)}\left[A^{\prime}(\xi)+\delta B(\xi)\right] \mathrm{d} \xi .
$$

From assumption $\mathrm{H}_{2}$, we obtain

$$
|Z(x)| \leq \frac{1}{\delta} k \int_{x}^{\bar{x}} \mathrm{e}^{-\delta|x-\xi|} \mathrm{e}^{-\eta \varphi(x)} \mathrm{e}^{\gamma|\xi|} \mathrm{d} \xi=\frac{k}{\delta} \int_{x}^{\bar{x}} \mathrm{e}^{-\delta|x-\xi|} \mathrm{e}^{-\eta \varphi(x)+\gamma|x|} \mathrm{e}^{\gamma(|\xi|-|x|)} \mathrm{d} \xi .
$$

From

we finally have

$$
|\xi|-|x| \leq|\xi-x| \text { and } \varphi(x) \geq|x|
$$

$$
|Z(x)| \leq \frac{k}{\delta} \int_{x}^{\bar{x}} \mathrm{e}^{-\delta|x-\xi|} \mathrm{e}^{(\gamma-\eta)|x|} \mathrm{e}^{\gamma|x-\xi|} \mathrm{d} \xi=\frac{k}{\delta} \int_{x}^{\bar{x}} \mathrm{e}^{(\gamma-\delta)|x-\xi|} \mathrm{e}^{(\gamma-\eta)|x|} \mathrm{d} \xi .
$$

This expression is bounded by the value at $\bar{x}$ or at $x_{0}$ depending on whether $\bar{x} \leq x_{0}$ or $\bar{x} \geq x_{0}$, because $\gamma-\delta<0$ and $\gamma-\eta<0$. Therefore $Z($.$) is bounded.$

Now we study the differentiability w.r.t. $x$ of the new function $W$ defined by

$$
W(x, \bar{\xi})=\mathrm{e}^{-\eta \varphi(x)} \frac{S(x, \bar{\xi})}{\delta} .
$$

When $x<\bar{\xi}$, we have

$$
W(x, \bar{\xi})=\frac{1}{\delta} \int_{x}^{\bar{\xi}} \mathrm{e}^{\delta(x-\xi)} \mathrm{e}^{-\eta \varphi(x)}\left[A^{\prime}(\xi)+\delta B(\xi)\right] \mathrm{d} \xi=\frac{\mathrm{e}^{\delta x} \mathrm{e}^{-\eta \varphi(x)}}{\delta} \int_{x}^{\bar{\xi}} \mathrm{e}^{-\delta \xi}\left[A^{\prime}(\xi)+\delta B(\xi)\right] \mathrm{d} \xi
$$

which is differentiable and whose derivative is given by:

$$
\frac{\partial W}{\partial x}(x, \bar{\xi})=\frac{\delta-\eta \varphi^{\prime}(x)}{\delta} \mathrm{e}^{\delta x-\eta \varphi(x)} \int_{x}^{\bar{\xi}} \mathrm{e}^{-\delta \xi}\left[A^{\prime}(\xi)+\delta B(\xi)\right] \mathrm{d} \xi-\frac{\mathrm{e}^{\delta x} \mathrm{e}^{-\eta \varphi(x)}}{\delta} \mathrm{e}^{-\delta x}\left[A^{\prime}(x)+\delta B(x)\right] .
$$


When $x>\bar{\xi}$, we have

$$
W(x, \bar{\xi})=\frac{1}{\delta} \int_{x}^{\bar{\xi}} \mathrm{e}^{-\delta(x-\xi)} \mathrm{e}^{-\eta \varphi(x)}\left[A^{\prime}(\xi)+\delta B(\xi)\right] \mathrm{d} \xi=\frac{\mathrm{e}^{-\delta x} \mathrm{e}^{-\eta \varphi(x)}}{\delta} \int_{x}^{\bar{\xi}} \mathrm{e}^{\delta \xi}\left[A^{\prime}(\xi)+\delta B(\xi)\right] \mathrm{d} \xi
$$

which is also differentiable, with:

$$
\frac{\partial W}{\partial x}(x, \bar{\xi})=\frac{-\delta-\eta \varphi^{\prime}(x)}{\delta} \mathrm{e}^{-\delta x-\eta \varphi(x)} \int_{x}^{\bar{\xi}} \mathrm{e}^{\delta \xi}\left[A^{\prime}(\xi)+\delta B(\xi)\right] \mathrm{d} \xi-\frac{\mathrm{e}^{-\delta x} \mathrm{e}^{-\eta \varphi(x)}}{\delta} \mathrm{e}^{\delta x}\left[A^{\prime}(x)+\delta B(x)\right] .
$$

At $x=\bar{\xi}$, we compute the following limits

$$
\lim _{x \rightarrow \bar{\xi}^{-}} \frac{\partial W}{\partial x}(x, \bar{\xi})=\lim _{x \rightarrow \bar{\xi}^{+}} \frac{\partial W}{\partial x}(x, \bar{\xi})=-\frac{\mathrm{e}^{-\eta \varphi(\bar{\xi})}}{\delta}\left(A^{\prime}(\xi)+\delta B(\xi)\right)
$$

therefore $W$ is also differentiable at $\bar{\xi}$. Moreover we observe that, for any $x$,

$$
\frac{\partial W}{\partial x}(x, \bar{\xi})=\frac{-\operatorname{sgn}(x-\bar{\xi}) \delta-\eta \varphi^{\prime}(x)}{\delta} \int_{x}^{\bar{\xi}} \mathrm{e}^{-\delta|x-\xi|} \mathrm{e}^{-\eta \varphi(x)}\left[A^{\prime}(\xi)+\delta B(\xi)\right] \mathrm{d} \xi-\frac{\mathrm{e}^{-\eta \varphi(x)}}{\delta}\left(A^{\prime}(x)+\delta B(x)\right)
$$

and we obtain

$$
\left|\frac{\partial W}{\partial x}(x, \bar{\xi})\right| \leq \frac{\delta+\eta}{\delta} \int_{x}^{\bar{\xi}} \mathrm{e}^{-\delta|x-\xi|} \mathrm{e}^{-\eta \varphi(x)} k \mathrm{e}^{\gamma|\xi|} \mathrm{d} \xi+\frac{\mathrm{e}^{-\eta \varphi(x)}}{\delta} k \mathrm{e}^{\gamma|x|} .
$$

This last expression is bounded because $\gamma-\eta<0$ and $\gamma-\delta<0$, the exponential being bounded by their values at $x$ or at $\bar{\xi}$ depending on whether $x \leq \bar{\xi}$ or $x \geq \bar{\xi}$.

Therefore we conclude that $W$ is uniformly continuous with respect to $x$. But $Z$ is given by

$$
Z(x)=\max _{\bar{\xi} \in E} W(x, \bar{\xi}), \quad \forall x \in \mathbb{R}
$$

which establishes that $Z$ is BUC.

Step 2. The function $Z($.$) is a viscosity solution of the Hamilton-Jacobi equation (8).$

From (26) we immediately obtain

$$
\frac{\partial W}{\partial x}(x, \bar{\xi})+\frac{1}{\delta} \mathrm{e}^{-\eta \varphi(x)}\left[A^{\prime}(x)+\delta B(x)\right]+\eta \varphi^{\prime}(x) W(x, \bar{\xi})=-\operatorname{sgn}(x-\bar{\xi}) \delta W(x, \bar{\xi}) .
$$

We now have to consider two cases:

(1) $\arg \max _{\bar{\xi}} W(x, \bar{\xi})=\{\bar{x}\}$;

(2) $\arg \max _{\bar{\xi}} W(x, \bar{\xi})$ not given by a single $\bar{x}$.

In the first case, at any $x$ such that $\arg \max _{\bar{\xi}} W(x, \bar{\xi})=\{\bar{x}\}$, the function $Z$ is differentiable and we have

$$
Z^{\prime}(x)=\frac{\partial W}{\partial x}(x, \bar{x})
$$

From (27) and (28), we deduce

$$
\left|Z^{\prime}(x)+\frac{1}{\delta} \mathrm{e}^{-\eta \varphi(x)}\left[A^{\prime}(x)+\delta B(x)\right]+\eta \varphi^{\prime}(x) Z(x)\right|=\delta|Z(x)|
$$

therefore $Z$ is an classical solution of the Hamilton-Jacobi equation (8) as soon as $Z(x) \geq 0$. 
In the second case: $Z$ is no more differentiable, and its sub-differentials are:

$$
\begin{aligned}
D^{+} Z(x) & =\emptyset \\
D^{-} Z(x) & =\overline{\operatorname{co}}\left\{\frac{\partial W}{\partial x}(x, \bar{x}), \bar{x} \in \arg \max _{\bar{\xi} \in E} W(x, \bar{\xi})\right\} \\
& =\left\{\sigma \delta Z(x)-\eta \varphi^{\prime}(x) Z(x)-\frac{\mathrm{e}^{-\eta \varphi(x)}}{\delta}\left[A^{\prime}(x)+\delta B(x)\right], \sigma \in[-1,1]\right\} .
\end{aligned}
$$

It then suffices to prove that $Z$ is a viscosity super-solution for the Hamilton-Jacobi equation (8). For $p^{-} \in D^{-} Z(x)$, we have

$$
\delta Z(x)-\left|p^{-}+\frac{1}{\delta} \mathrm{e}^{-\eta \varphi(x)}\left[A^{\prime}(x)+\delta B(x)\right]+\eta \varphi^{\prime}(x) Z(x)\right|=\delta Z(x)(1-\sigma)
$$

which is non negative for all $\sigma \in[-1,1]$ if and only if $Z(x) \geq 0$.

We can now easily deduce from this proposition the well known sufficient condition in the turnpike literature (2), when the function $C$ has only one change of sign (see point 1 of the corollary below). Furthermore, we show that the usual sufficient condition, which is required to be fulfilled globally, is also a necessary condition but only locally (see point 2 of the corollary).

Corollary 4.2. Assume $\left(H_{1}\right)-\left(H_{2}\right)$,

1. If the function $C($.$) has exactly one zero \bar{x}$ on $\mathbb{R}$ and fulfills the property.

$$
C(x)(\bar{x}-x) \geq 0
$$

at any $x \in \mathbb{R}$, then the most rapid approach path to $\bar{x}$ is optimal from any initial condition $x_{0} \in \mathbb{R}$;

2. For any $\bar{x} \in E$ such that $\mathcal{B}(\bar{x})$ is non empty, there exists a neighborhood $\mathcal{V}$ of $\bar{x}$ such that the property (29) is fulfilled on $\mathcal{V}$.

Proof.

1. When $x<\bar{x}, C(x)$ is positive and thus $S(x, \bar{x})$ is also positive. For $x=\bar{x}$, one has $S(\bar{x}, \bar{x})=0$. When $x>\bar{x}, C(x)$ is negative and thus $S(x, \bar{x})$ is positive. So, $\bar{S}(x)=S(x, \bar{x})$ is always non negative, and we conclude by Proposition 4.1 that the $\operatorname{MRAP}\left(x_{0}, \bar{x}\right)$ is optimal for any $x_{0} \in \mathbb{R}$.

2. Assume that $\mathcal{B}(\bar{x})$ is non empty. We first show that $\bar{x}$ necessarily belongs to it. Take any $x_{0} \in \mathcal{B}(\bar{x})$, then the $\operatorname{MRAP}\left(x_{0}, \bar{x}\right)$ is an optimal trajectory that reaches $\bar{x}$ at a finite time, say at $\tau$. Then, applying Bellman's principle, the stationary trajectory $\bar{x}($.$) is optimal from the initial condition x(\tau)=\bar{x}$, so $\bar{x} \in \mathcal{B}(\bar{x})$.

Consider the set $I:=\left\{x<\bar{x} \mid S_{x}(x, \bar{x})>0\right\} \cup\left\{x>\bar{x} \mid S_{x}(x, \bar{x})<0\right\}$. The expression of $S_{x}(x, \bar{x})$ :

$$
S_{x}(x, \bar{x})=\mid \begin{aligned}
& -C(x)+\delta S(x, \bar{x}) \text { if } x \leq \bar{x} \\
& -C(x)-\delta S(x, \bar{x}) \text { if } x \geq \bar{x}
\end{aligned}
$$

gives:

$$
I=\{x<\bar{x} \mid-C(x)+\delta S(x, \bar{x})>0\} \cup\{x>\bar{x} \mid-C(x)-\delta S(x, \bar{x})<0\} .
$$

The functions $x \mapsto C(x)$ and $x \mapsto S(x, \bar{x})$ being continuous, we deduce that $I$ is open set. Let us show that $\bar{x}$ cannot belong to the closure of $I$. If it does, then

- either there exists $\bar{x}^{-}<\bar{x}$ such that $S_{x}(., \bar{x})$ is positive on $] \bar{x}^{-}, \bar{x}[$,

- either there exists $\bar{x}^{+}>\bar{x}$ such that $S_{x}(., \bar{x})$ is negative on $] \bar{x}, \bar{x}^{+}[$. 
Assume for instance that the first case is verified (the treatment of the second case is similar). Recall that $S(\bar{x}, \bar{x})=0$, then one has $S(., \bar{x})<0$ on $] \bar{x}^{-}, \bar{x}[$. Consider then a trajectory $\widetilde{x}(.) \in \operatorname{Adm}(\bar{x})$ which is a $\operatorname{MRAP}(\bar{x}, \xi)$ for $\xi \in] \bar{x}^{-}, \bar{x}[$, and write

$$
\begin{aligned}
J[\widetilde{x}(.)] & =\frac{A(\bar{x})}{\delta}+\frac{1}{\delta} \int_{\bar{x}}^{\xi} \mathrm{e}^{-\delta(y-\bar{x})} C(y) \mathrm{d} y \\
& =\frac{A(\bar{x})}{\delta}+\mathrm{e}^{\delta(\bar{x}-\xi)} \int_{\bar{x}}^{\xi} \mathrm{e}^{\delta(\xi-y)} C(y) \mathrm{d} y \\
& =\frac{A(\bar{x})}{\delta}-\mathrm{e}^{\delta(\bar{x}-\xi)} S(\xi, \bar{x})>\frac{A(\bar{x})}{\delta}
\end{aligned}
$$

thus a contradiction with the optimality of the stationary trajectory $\bar{x}($.$) , for which J[\bar{x}()]=.A(\bar{x}) / \delta$. So, $\bar{x}$ does not belong to $I$, thus the existence of a neighborhood $\mathcal{V}$ of $\bar{x}$ such that

$$
\forall x \in \mathcal{V},\left\{\begin{array}{l}
x \leq \bar{x} \Rightarrow S_{x}(x, \bar{x}) \leq 0 \Rightarrow S(x, \bar{x}) \geq 0 \Rightarrow C(x)=\delta S(x, \bar{x})-S_{x}(x, \bar{x}) \geq 0 \\
x \geq \bar{x} \Rightarrow S_{x}(x, \bar{x}) \geq 0 \Rightarrow S(x, \bar{x}) \leq 0 \Rightarrow C(x)=-\delta S(x, \bar{x})-S_{x}(x, \bar{x}) \leq 0
\end{array}\right.
$$

which exactly implies that the condition $(29)$ is fulfilled on $\mathcal{V}$.

\section{An eXAmple}

We now give an example for which the Euler equation is singular and admits more than one stationary solutions, none of them satisfying (2), or (29) everywhere. From the condition (20), we deduce the optimality of $\operatorname{MRAP}\left(x_{0}, \bar{x}\right)$ for one or several turnpikes.

Let $0<a<b$ and consider the problem:

$$
\max _{x(.)} \int_{0}^{\rightarrow \infty} \mathrm{e}^{-t} x^{2}(t)[2 \dot{x}(t)(a+b-x(t))-a b] \mathrm{d} t \text { with } \dot{x}(t) \in[-1,+1] \text { a.e. }
$$

In this example we have

$$
A(x)=-a b x^{2}, \quad B(x)=2 x^{2}(a+b-x) \quad \text { and } \quad \delta=1 .
$$

Then the Euler equation is:

$$
C(x)=-2 x\left(a b+(a+b) x-x^{2}\right)=-2 x(a-x)(b-x),
$$

which admits three solutions: $\bar{x} \in\{0, a, b\}$ (see Fig. 1).

We observe that $\bar{x}=0$ and $\bar{x}=b$ satisfies (only locally) the classical condition (29). From Proposition 4.1, we prove now that depending on the values of $a$ and $b$ :

- $\operatorname{MRAP}\left(x_{0}, 0\right)$ or $M R A P\left(x_{0}, b\right)$ is optimal for any initial condition $x_{0}$;

- there exists $\left.x^{*} \in\right] 0, b\left[\right.$ such that $M R A P\left(x_{0}, 0\right)\left(\operatorname{resp} . M R A P\left(x_{0}, b\right)\right)$ is optimal for $x_{0} \leq x^{*}\left(\operatorname{resp} . x_{0} \geq x^{*}\right)$.

It is easy to verify the following properties:

$$
\left\{\begin{array}{l}
x \in[0, b] \Rightarrow S(x, a) \leq 0 \\
x \leq a \Rightarrow\{S(x, 0) \geq 0 \text { et } S(x, b) \geq S(0, b)\} \\
x \geq b \Rightarrow S(x, 0) \geq S(b, 0) \\
x \geq a \Rightarrow S(x, b) \geq 0 .
\end{array}\right.
$$




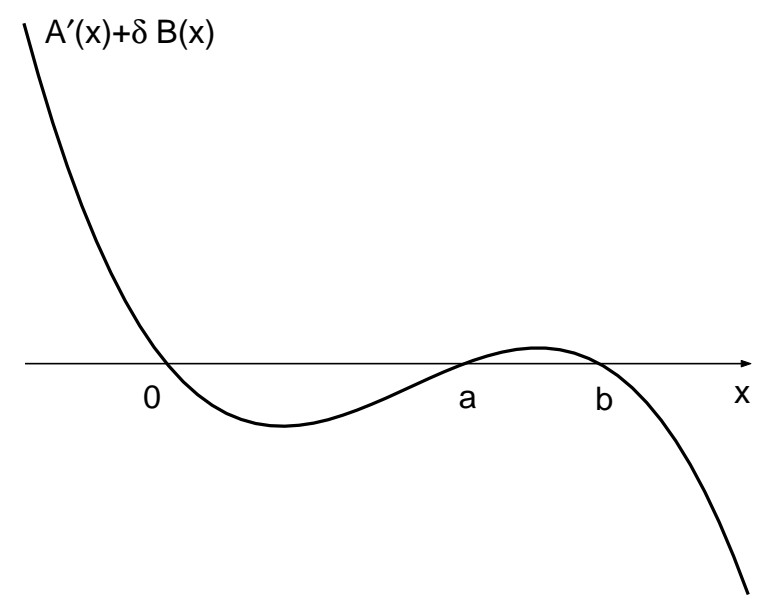

FiguRE 1. The function $x \mapsto C(x)$.

Therefore we can conclude by Proposition 4.1 and Corollary 4.2 that:

1. $\operatorname{MRAP}\left(x_{0}, a\right)$ is never optimal for any initial condition $x_{0}$;

2. $\operatorname{MRAP}\left(x_{0}, 0\right)$ is optimal for all $x_{0}$ as soon as $S(b, 0) \geq 0$;

3. $\operatorname{MRAP}\left(x_{0}, b\right)$ is optimal for all $x_{0}$ as soon as $S(0, b) \geq 0$;

4. If $x \rightarrow \max \{S(x, 0), s(x, b)\}$ is non negative for all $x \in[0, b]$, then $M R A P\left(x_{0}, 0\right)$ or $M R A P\left(x_{0}, b\right)$ is optimal.

For $x \in[0, b]$, we compute the following functions

$$
S(x, 0)=\mathrm{e}^{-x} \int_{0}^{x} 2 y(a-y)(b-y) \mathrm{e}^{y} \mathrm{~d} y \quad \text { et } \quad S(x, b)=-\mathrm{e}^{x} \int_{x}^{b} 2 y(a-y)(b-y) \mathrm{e}^{-y} \mathrm{~d} y,
$$

with the help of a symbolic computation software:

$$
\left\{\begin{aligned}
S(x, 0)= & 2\left[x^{3}-(a+b+3) x^{2}+(a b+2(a+b+3))\left(x-1+\mathrm{e}^{-x}\right)\right] \\
S(x, b)= & 2\left[-x^{3}+(a+b-3) x^{2}+(-a b+2(a+b-3))\left(x+1+\mathrm{e}^{x-b}\right)\right. \\
& \left.+\left(b^{2}-4 a+2 b+12\right) \mathrm{e}^{x-b}\right] .
\end{aligned}\right.
$$

Then for different values of $a$ et $b$, we obtain the three following possible cases:

1. For $a=2$ et $b=3$, we have

$$
S(b, 0)=2\left(22 \mathrm{e}^{-3}-1\right)>0
$$

and we can conclude that the paths that are going with a most rapid velocity to $\bar{x}=0$ are optimal (see Fig. 2); 2. For $a=1$ et $b=4$, we obtain

$$
S(0, b)=64 \mathrm{e}^{-4}>0
$$

and we can conclude that the paths that are going with a most rapid velocity to $\bar{x}=b$ are optimal (see Fig. 3 ); 3. For $a=2$ et $b=5$, we obtain

$$
\left\{\begin{array}{l}
S(b, 0)=2\left(30 \mathrm{e}^{-5}-5\right)<0 \\
S(0, b)=2\left(37 \mathrm{e}^{-5}-2\right)<0
\end{array}\right.
$$

but we observe that $\max \{S(x, 0), s(x, b)\}$ is non negative on $[0, b]$. Let $\left.x^{*} \in\right] 0, b\left[\right.$ be such that $S\left(x^{*}, 0\right)=S\left(x^{*}, b\right)$ (see Fig. 4). Then there is a competition between the two turnpikes $\bar{x}=0$ and $\bar{x}=b$ : for $x_{0} \leq x^{*}\left(\right.$ resp. $\left.x_{0} \geq x^{*}\right)$, it is optimal to go as quickly as possible to $\bar{x}=0$ (resp. $\bar{x}=b$ ). Let us underline that in this last case there 


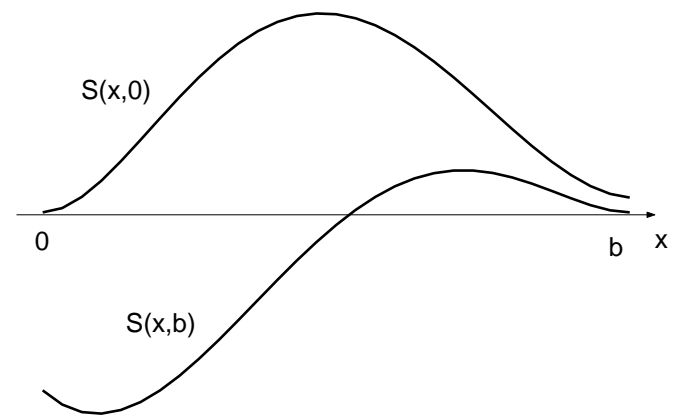

Figure 2. Functions $x \mapsto S(x, 0)$ and $x \mapsto S(x, b)$ for $a=2$ and $b=3$.

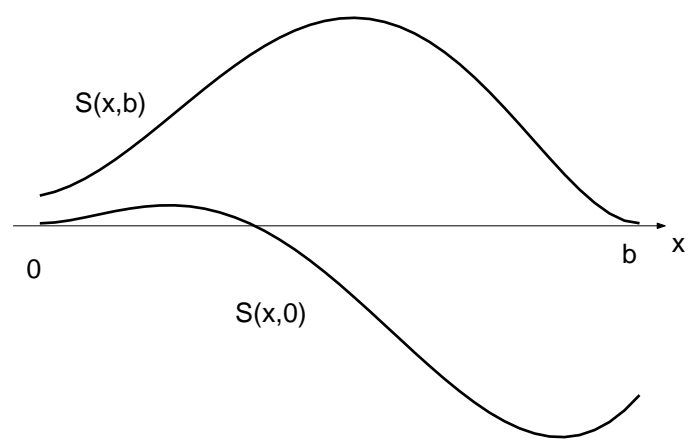

Figure 3. Functions $x \mapsto S(x, 0)$ and $x \mapsto S(x, b)$ for $a=1$ and $b=4$.

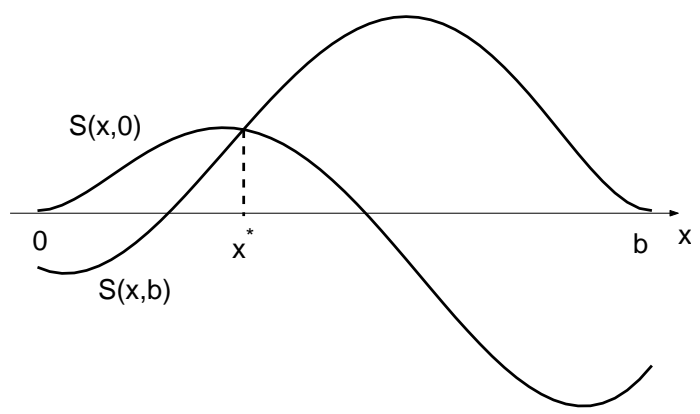

Figure 4. Functions $x \mapsto S(x, 0)$ and $x \mapsto S(x, b)$ for $a=2$ and $b=5$.

is no longer uniqueness of the turnpike for the initial condition $x_{0}=x^{*}$, which also corresponds to a nondifferentiability point of the value function.

\section{Conclusion}

For singular scalar problems of calculus of variation in infinite horizon, we have proposed a new necessary and sufficient condition for the optimality of MRAPs. This condition generalizes the standard one which is only sufficient and valid only in the case of a unique solution of the singular Euler equation. Our result is established with the characterization of a transformation of the value function, in terms of viscosity solutions of a particular Hamilton-Jacobi, which provides global optimality conditions. Our condition also applies when one is dealing 
with singular Euler equations that possess more than one solutions. The lack of differentiability of the value function is connected to the existence of competition between most rapid approaches towards several singular arcs.

Acknowledgements. This work has been accomplished during the stay of P. Cartigny at the LASB (Laboratoire d'Analyse des Systèmes et de Biométrie), Montpellier. He is grateful to INRA (Institut National de la Recherche Agronomique) for his grant.

\section{REFERENCES}

[1] G. Barles, Solutions de viscosité des équations de Hamilton-Jacobi. Springer-Verlag, Paris-Heidelberg-New York (1994).

[2] D.J. Bell and D.H. Jacobson, Singular Optimal Control Problems. Academic Press, London (1975).

[3] J. Blot and P. Cartigny, Optimality in Infinite Horizon Variational Problems under Signs Conditions. J. Optim. Theory Appl. 106 (2000) 411-419.

[4] J. Blot and P. Michel, First-Order Necessary Conditions for the Infinite-Horizon Variational Problems. J. Optim. Theory Appl. 88 (1996) 339-364.

[5] P. Cartigny and P. Michel, On a Sufficient Transversality Condition for Infinite Horizon Optimal Control Problems. Automatica 39 (2003) 1007-1010.

[6] C.W. Clark, Mathematical Bioeconomics: The Optimal Management of Renewable Resources. Wiley, New York (1976).

[7] I. Ekeland, Some Variational Problems Arising from Mathematical Economics. Springer-Verlag, Lecture Notes in Math. 1330 (1986).

[8] R.F. Hartl and G. Feichtinger, A New Sufficient Condition for Most Rapid Approach Paths. J. Optim. Theory Appl. 54 (1987).

[9] M.G. Crandall and P.-L. Lions, Viscosity Solutions of Hamilton-Jacobi Equations. Trans. Americ. Math. 277 (1983) 1-42.

[10] A. Miele, Extremization of Linear Integrals by Green's Theorem, Optimization Technics, G. Leitmann Ed. Academic Press, New York (1962) 69-98.

[11] A. Rapaport and P. Cartigny, Théorème de l'autoroute et équation d'Hamilton-Jacobi. C.R. Acad. Sci. 335 (2002) $1091-1094$. 